OPEN ACCESS

Edited by:

Andrey R. Nikolaev,

Lund University, Sweden

Reviewed by:

Bertram Emil Shi,

Hong Kong University of Science and Technology, Hong Kong

Agostino Gibaldi,

University of California, Berkeley,

United States

*Correspondence:

Jacek Tursk

turskij@gmail.com

${ }^{\dagger}$ Professor Emeritus

Specialty section:

This article was submitted to

Perception Science,

a section of the journal

Frontiers in Neuroscience

Received: 27 April 2020 Accepted: 18 September 2020 Published: 07 December 2020

Citation:

Turski J (2020) A Geometric Theory Integrating Human Binocular Vision With Eye Movement.

Front. Neurosci. 14:555965. doi: 10.3389/fnins.2020.555965

\section{A Geometric Theory Integrating Human Binocular Vision With Eye Movement}

\author{
Jacek Turski ${ }^{*+}$ \\ Department of Mathematics and Statistics, University of Houston-Downtown, Houston, TX, United States
}

A theory of the binocular system with asymmetric eyes (AEs) is developed in the framework of bicentric perspective projections. The AE accounts for the eyeball's global asymmetry produced by the foveal displacement from the posterior pole, the main source of the eye's optical aberrations, and the crystalline lens' tilt countering some of these aberrations. In this theory, the horopter curves, which specify retinal correspondence of binocular single vision, are conic sections resembling empirical horopters. This advances the classic model of empirical horopters as conic sections introduced in an ad hoc way by Ogle in 1932. In contrast to Ogle's theory, here, anatomically supported horopteric conics vary with the AEs' position in the visual plane of bifoveal fixations and their transformations are visualized in a computer simulation. Integrating horopteric conics with eye movements can help design algorithms for maintaining a stable perceptual world from visual information captured by a mobile robot's camera head. Further, this paper proposes a neurophysiologically meaningful definition for the eyes' primary position, a concept which has remained elusive despite its theoretical importance to oculomotor research. Finally, because the horopteric conic's shape is dependent on the AE's parameters, this theory allows for changes in retinal correspondence, which is usually considered preformed and stable.

\footnotetext{
Keywords: binocular vision, eye movement, retinal correspondence, horopter, eye's aplanatic design, asymmetric model eye, conic sections, vergence resting position
}

\section{INTRODUCTION}

Our eyes receive two disparate perspective projections of a scene due to their bilateral separation. Their two-dimensional (2D) layer of photoreceptors sampling these projections is part of an unstable retinal circuitry. This happens because our eyes are constantly moving 3-4 times per second to fixate the high-acuity fovea successively on the salient and behaviorally relevant parts of the scene (Kowler, 2011). Thus, there is visible motion due to eye movements even during steady fixations (Martinez-Conde et al., 2004).

Therefore, the retinal images have visible motion due to both the eyes' incessant movements and the movements of objects in the scene. Although this should lead to a compromised understanding of the scene, we instead perceive, with vivid impressions of forms in depth, stable visual scenes containing moving objects. To understand our perceived constancy of a 3D world from 2D, unstable sensory inputs, we need to understand how binocular vision is integrated with the eyes' movements. 
Whenever a retinal element is stimulated by a localized light, the stimulus is perceived in a specific direction. If the stimulus projecting to the two retinal elements, one for each eye in the binocular system, is perceived in the same direction, then they are considered to be corresponding elements. Normal correspondence occurs when the fovea of one eye corresponds to the fovea of the other eye; their single visual direction is called the principal visual direction, or the Cyclopean direction. The visual directions of all other pairs of stimulated corresponding elements are perceived in relation to this principal direction. The horopter is the set of all points in the binocular visual field stimulating retinal corresponding elements. Because the normal binocular vision is specified by two foveae being corresponding, all other corresponding retinal elements can then be determined from laboratory measurements of the empirical horopter (Ogle, 1950; Shipley and Rawlings, 1970).

The empirical horopters were comprehensively modeled in Ogle (1932) and Amigo (1965) and, more recently, in Turski (2016c, 2018). The equations with free parameters that were introduced on an ad hoc basis in Ogle (1932) for the forward gaze and extended in Amigo (1965) to any horizontal gaze furnished longitudinal horopters as conic sections. Introduced in Turski (2016c) and numerically studied in Turski (2018), the empirical horopters were modeled as conic sections in the binocular system with asymmetric eyes (AEs). The AE is the model eye that extends the reduced eye with its inclusion of the fovea's displacement from the posterior pole and the cornea's and lens' relative tilts observed in healthy human eyes (Chang et al., 2007; Schaeffel, 2008). This fovea's anatomical displacement is the main source of optical aberrations and the lens' tilts cancel out some of these aberrations by contributing to the eye's aplanatic design (Tabernero et al., 2007; Artal, 2014).

My studies in Turski (2018) found that the horopteric conics were numerically similar but geometrically different from the conic sections in Ogle (1932) and Amigo (1965); my conic sections pass through the nodal points' anatomical location and their conic sections pass, incorrectly, through the eyes' rotation centers. Further, in my studies, the straight-line empirical horopter, defining the abathic distance to the symmetrically fixated point, resulted from the AEs' position in which their lens' equatorial planes are coplanar. Then, when the AE's parameters are set to the average values for the human eye, the resulting abathic distance of $1 \mathrm{~m}$ complies with its average physiological value in humans (Gibaldi et al., 2017). This resulting abathic distance is also within the range of the eye muscles' natural tonus resting position distance (Jaschinski-Kruza, 1991; Jaschinski et al., 2007).

In this paper, I extend numerical studies in Turski (2018) by developing a simple geometric theory in which the retinal correspondence of the binocular system with AEs is elaborated in the framework of bicentric perspective projections (Koenderink, 1992). Because the eye muscles' natural tonus resting position serves as a zero-reference level for convergence effort (Ebenholtz, 2001), this theory contends that the primary position of the AEs coincides with the abathic-distance bifoveal fixation. The primary position, originally intended for a single eye, is often described in binocular vision as both eyes being directed straight ahead by an erect head. This rather imprecise definition of the eyes' primary position could be the reason for its neurophysiological significance remaining elusive despite its theoretical importance to oculomotor research (Hess and Thomassen, 2014). Thus, this novel characterization of the eyes' primary position integrates binocular conics with the eyes' movements in a precise and natural way that has been unavailable until now.

The result of such an integration is that we are now able to graphically simulate the horopteric conics' transformations from the movement of the fixation point in the visual plane, which also demonstrate the horopteric conics' classification in terms of the eyes' position. GeoGebra's dynamic geometry software is used in this paper to demonstrate all geometric results found for the horopters and the retinal correspondence. The simulation of horopteric conics' transformations is included in the Supplementary Material.

The theory's binocular framework of bicentric retinal projections accounts for the fact that the human decodes properties of the $3 \mathrm{D}$ environment from neural processes fundamentally constrained by the sensory organs' geometric relationships to the environment (Rokers et al., 2011; Bonnen et al., 2019). In addition, the $\mathrm{AE}$ accounts for some of the eye's aplanatic design that correlates the lens's misalignment with corneal aberration to produce nearly diffraction-free retinal images close to the visual axis (Artal, 2014).

Although the distribution of retinal corresponding elements is usually considered fixed (Hillis and Banks, 2001), the horopter's shape and the retinal correspondence are dependent on the asymmetry parameters of the model eye and can, therefore, change when the AE's parameters change. For example, when the crystalline lens is replaced during refractive surgery with a toric intraocular lens (IOL), it does not only correct for refractive errors and provide sharper focus, but corneal astigmatism can be also be corrected for by adjusting the lens's orientation. The evaluation of a group of patients in Wang et al. (2019) shows that the IOL tilt magnitude increased significantly compared to the preoperative crystalline lens's tilt. This increase in tilt can postoperatively modify the horopter's shape and the retinal correspondence.

\section{ASYMMETRIC EYE}

The AE model (Figure 1), discussed in detail in Turski (2018), incorporates the most important features of the human eye's asymmetric design. However, the AE model is slightly modified here by its use of the effective lens. The eye's natural asymmetry is modeled by two parameters; the angle $\alpha$ which specifies the fovea's temporalward displacement from the posterior pole, and the angle $\beta$ which gives the effective lens' tilt and decentration relative to the optical axis. The effective lens introduced in the AE model simplifies the description of the lens' tilt and defines the optical axis as the eyeball's line of axial symmetry when $\alpha=$ $\beta=0$. I assume that $\alpha-\beta>0$ because it is satisfied in a typical binocular system. Because angle $\alpha$ has a low interpatient variability (Holladay, 2007), I use the angle $\alpha$ 's average value of 


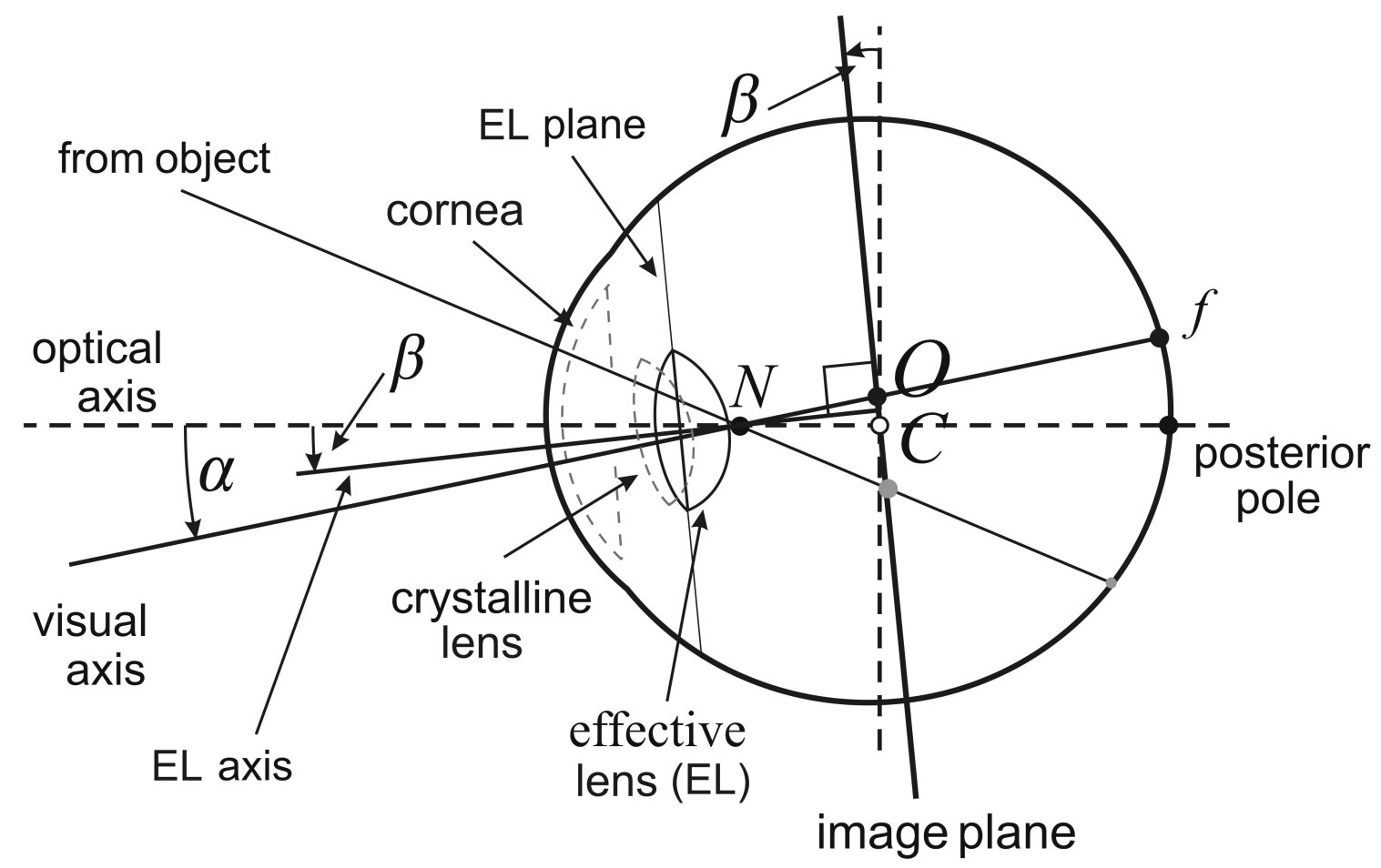

FIGURE 1 | Asymmetric eye (AE) model for the right eye. The fovea, $f$, is displaced from the posterior pole by the eyeball's global tilt of $\alpha$ degrees. The relative misalignment of the cornea and lens is represented by the $\beta$-degree tilt of the equatorial plane of a single effective lens. Both angles of tilt are at the nodal point $N$ located on the optical axis $0.6 \mathrm{~cm}$ anterior to the eyeball's rotation center $C$. The optical axis is defined by $\alpha=\beta=0$. The image plane is obtained by tilting the frontal plane by $\beta$ degrees at the eyeball's rotation center $C$. The visual axis passing through $N$ and $f$ intersects the image plane at its optical center $O$.

$5.2^{\circ}$. Angle $\beta$ is assumed to vary between $-0.4^{\circ}$ and $4.7^{\circ}$, the range of $\beta$ 's values observed in human eyes.

The tilt of the effective lens is represented in my geometrical model of the binocular system with AEs by the image plane passing through the eye's rotation center that is parallel to the equatorial plane of the effective lens. The image impinged on the retina is defined by the pencil of light rays passing through the nodal point. In the $\mathrm{AE}$ model, these light rays may be parameterized in angular coordinates on the curved surface of the retina, or on the image plane with more convenient in image processing rectangular coordinates. The cornea and crystalline lens's misalignment, represented by the effective lens' tilt, is one of the eye's sophisticated aplanatic elements designed to compensate for some of the limitations to optical quality caused by the fovea's displacement form the eyeball's posterior pole (Artal, 2014).

\section{BINOCULAR SYSTEM WITH AES}

The horopteric conic sections resembling empirical horopters were numerically studied in Turski (2018) in the binocular system with AEs. This section introduces basic definitions in the geometry of this binocular system displayed in Figure 2, but a detailed elaboration of binocular geometry is developed throughout the following sections. In particular, the horopteric curves and retinal correspondence are geometrically constructed in the next two sections.
I note that in the binocular system with symmetric eyes, i.e., with model eyes satisfying $\alpha=\beta=0, \phi_{r}$ and $\phi_{l}$ are angles describing the eyes' rotations from their primary position, often described as both eyes directed straight ahead in an erect head. In this case, the angle subtended at the resulting fixation point is given by the vergence angle

$$
\eta=\phi_{r}-\phi_{l}
$$

see fixation $F_{0}$ in Figure 3C.

The distance to the symmetrically fixated point, $F_{a}$ shown in Figure 2, through which a linear horopter is passing, is known as the abathic distance. To get the subtense of the fixation points $F_{a}$ and another point $F$, I use the equality of alternate angles: two angles, not adjoined, formed on opposite sides of a line where the line intersects two other parallel lines. In Figure $2, \alpha-\beta-\phi_{l}$ at both vertices, $N_{l}$ and $F$, are alternate angles for the left eye, and $\alpha-\beta+\phi_{r}$ at both vertices, $N_{r}$ and $F$, are alternate angles for the right eye. Note that the angle $\phi_{l}$ is subtracted from $\alpha-\beta$ because its value is negative. It is easy then to verify, by taking the sums of respective alternate angles, that the angle subtended by visual lines at $F_{a}$ is $2(\alpha-\beta)$ and the angle at $F$ is $2(\alpha-\beta)+\eta$. Thus, since $\alpha \neq \beta$, the angle at any fixation obtained by the change of gaze from $F_{a}$ never takes on the vergence angle $\eta$ in Equation (1). Therefore, in this work, the angles subtended by the visual lines at the spatial points are called binocular subtense, or just subtense. 


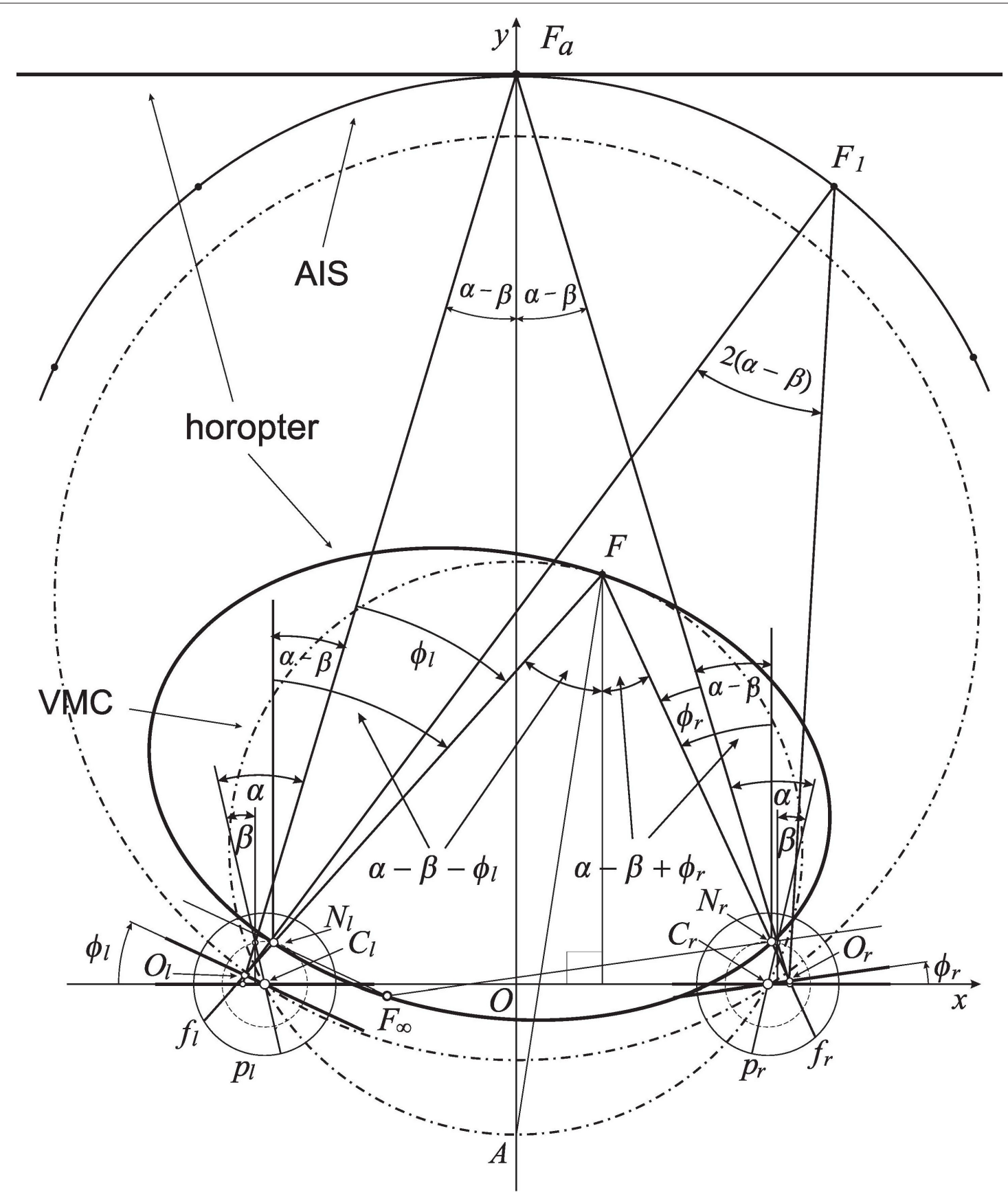

FIGURE 2 | The eyes' asymmetry angles $\alpha$ and $\beta$ are shown only for fixation $F_{a}$. The rotating angles, $\phi_{r}$ and $\phi_{l}$, change the eyes' gaze from the abathic-distance position, fixation $F_{a}$, to the position in which eyes are fixating on $F$. This results in the subtense changing from $\sigma_{a}=2(\alpha-\beta)$ at $F_{a}$ to the subtense $\sigma_{F}=2(\alpha-\beta)+\eta$ at $F$, where $\eta$ is the vergence (1). The horopteric ellipse, shown here for the fixation $F$, is constructed in section 4 using the nodal points, $N_{r}$ and $N_{l}$, and the intersection point, $F_{\infty}$, of the lines through the nodal points and parallel to the respective image planes. The condition $\phi_{r}-\phi_{l}=0$ furnishes a curve with a constant subtense $\sigma_{a}$. This is the abathic iso-subtense curve (AIS) that passes through $F_{a}$. Later, similarly to the case of the symmetric (reduced) model eye, the Cyclopean direction of the fixation point $F$ in the binocular system with AEs will be specified relative to the point $A$ on the Vieth-Müller circle (VMC) passing through $F$.

Eye positions reached from the abathic distance fixation by equal eye rotations, $\phi_{r}=\phi_{l}$, have fixation points that lie on, what I call, the abathic iso-subtense curve (AIS). For each different symmetric fixation of subtense $2(\alpha-\beta)+\eta$, we get different iso-subtense curve. This curves differ from the iso-vergence curves, or Vieth-Müller circles (VMCs), because, in contrast to the iso-subtense curves, the VMC passes through the eyes' rotation centers. The AIS curve, the iso-subtense curve which 


\section{A}

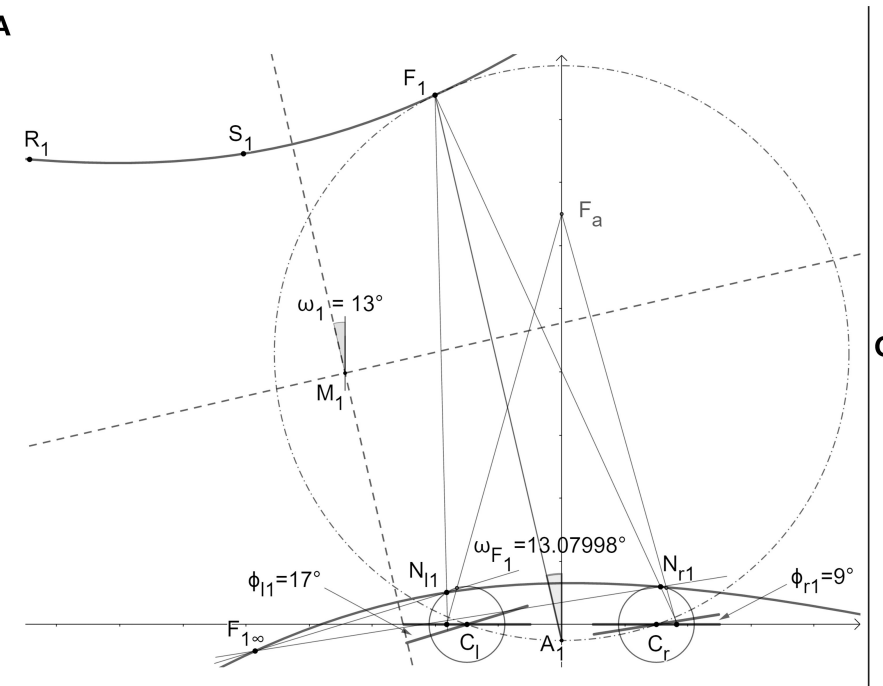

\section{B}

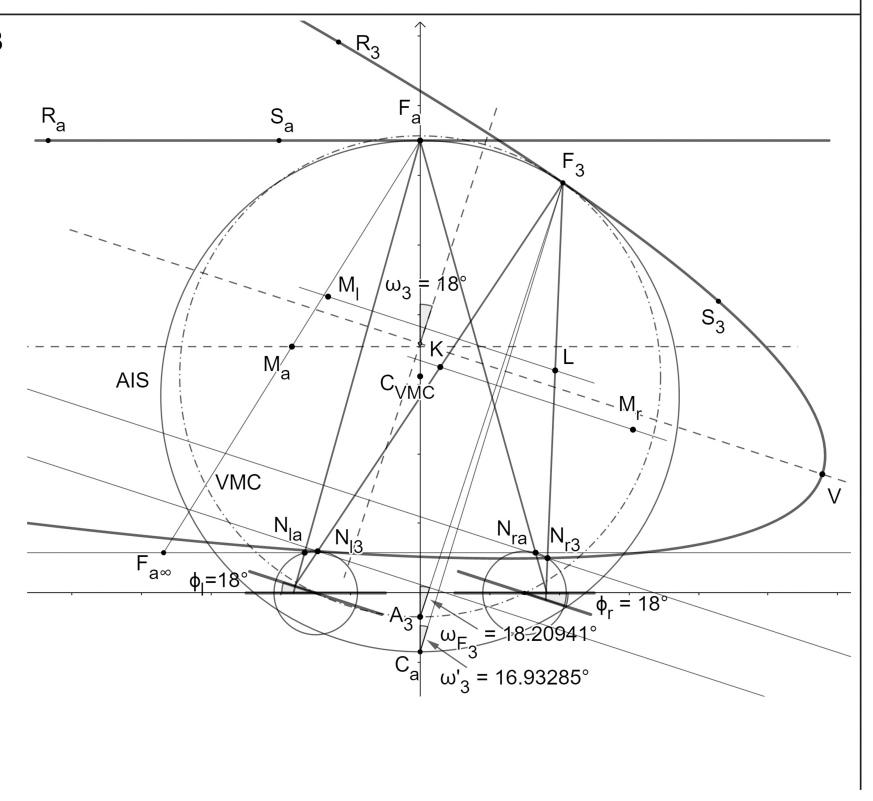

C
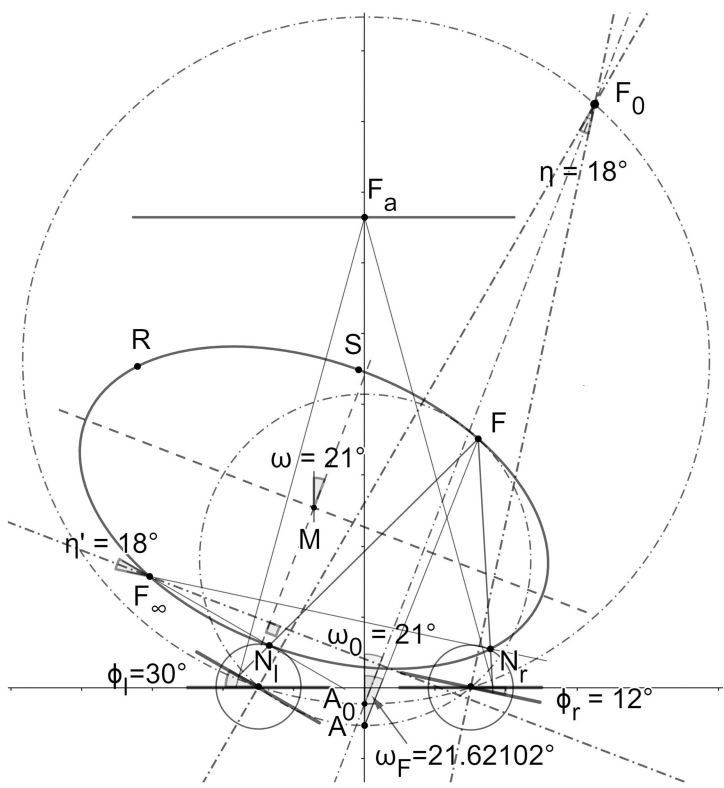

FIGURE 3 | The horopteric conic sections constructed in the demonstration of BINOCULAR CONICS CONSTRUCTION. (A) The hyperbola and the ellipse (not shown). (B) The straight line and the parabola. (C) The proof that the conic section orientation is given by version angle, carried out only for the ellipse.

passes through $F_{a}$ at the abathic distance, is graphed numerically in Figure 2 for fixations in the azimuthal range $\pm 45^{\circ}$, the neurally determined range of typical gaze eccentricities (Guitton and Volle, 1987). For anthropomorphic binocular system parameters, the AIS will be closely approximated in section 6 by the VMC.

Further, for symmetric eyes $(\alpha=\beta=0)$, the version angle,

$$
\omega=(1 / 2)\left(\phi_{r}+\phi_{l}\right)
$$

is the azimuthal angle of the ray that starts from the point on the VMC's that is midway between the eyes' centers and passes through the fixation point (cf. the fixation point $F_{0}$ in Figure 3C) given by the rotation angles $\phi_{r}$ and $\phi_{l}$ from the eyes' primary position. I recall that the eyes' primary position is often described as both eyes directed straight ahead in an erect head. The VMC's midpoint and the azimuthal angle (2) specify the Cyclopean eye's position (Turski, 2016a) and, hence, the principal visual direction. Section 6 will discuss how the fixation point F's Cyclopean eye position can be defined in the binocular system with AEs.

\section{THE GEOMETRIC CONSTRUCTION OF BINOCULAR CONICS}

In the binocular system with AEs, parameters $\alpha, \beta, a$, and the eyes' rotation angles $\phi_{r}$ and $\phi_{l}$ specify coordinates of the four points in the horizontal visual plane which lie on, or 
are associated with, the corresponding horopteric curve. These points are the nodal points $N_{r}$ and $N_{l}$, the fixation point $F$, and the point denoted by $F_{\infty}$. The point $F_{\infty}$ (cf. Figure 2) is the intersection of the two lines, each passing through the nodal point of one eye and parallel to both the AE's image plane and effective lens' equatorial plane. Thus, in the projective geometry framework (Henle, 1997), $F_{\infty}$ projects to the points at infinity, one for each of the AE's image planes. The fixation point $F$ projects along the visual axes to the foveae, which are corresponding retinal elements in normal binocular vision. In contrast, the lines projected from $F_{\infty}$ to the pair of points at infinity do not intersect the retinae.

Thus, the points at infinity are not corresponding retinal elements though they are called geometrical corresponding points here because of the significant role they play in the horopteric curves' geometric constructions. These geometrical constructions for the binocular system with AEs are given below in this section. These constructions are motivated by the results obtained in Turski (2016a) for horopteric circles in binocular system with symmetric (reduced) eyes. They are reframed here in Proposition 1 to include $F_{\infty}$ into the formulation, which is otherwise not needed because it is a theorem of Euclidean geometry.

Proposition 1. Let the nodal point be located on the optical axis at any point at or between the spherical eyeball's rotation center and its pupil. Then, for the binocular eyes' position with fixation point $F$ in the horizontal visual plane, the lines that pass through the nodal points and are perpendicular to the visual axes intersect at the point $F_{\infty}$ on the circular horopter. It then follows that line segment $F F_{\infty}$ must pass through the horopteric circle's center.

The proof of Proposition 1 is given in Supplement 1. It shows that, in the binocular system with symmetric model eyes, $F$ and $F_{\infty}$ are diagonally opposite points on the horopteric circle. The anatomically correct location of the nodal point is $0.6 \mathrm{~cm}$ anterior to the eye's rotation center, though the proof is for any nodal point location between the eye's rotation center and pupil.

The construction of horopteric curves in binocular system with AEs incorporates the horopteric circles' point symmetry of Proposition 1. The rationale for this extension is the continuity requirement of the horopteric curves' transformations as the AE's parameters $\alpha$ and $\beta$ both approach zero. Moreover, referring to my previous research, the extension also accurately reflects $F$ and $F_{\infty}$ 's relation in projective geometry, the geometric framework that is essential to the constructions of horopteric curves for the binocular system with AEs.

To explicate this further, I note that the mapping between points of the spherical retina and points of the image plane can be modeled by stereographic projection through the nodal point for both symmetric and asymmetric model eyes (Turski, 2016c). This mapping is not defined at the nodal point. Stereographic projection is extended to one-to-one and onto by appending the image of the nodal point under the mapping, called the point at infinity, to the image plane. The image plane with the point at infinity is the celebrated object in geometry and mathematical analysis known as the Riemann sphere (Needham,
2002). Stereographic projection is conformal, that is, it preserves the angle of two intersecting curves. Further, it maps circles in the spherical retina that do not contain the nodal point to circles in the image plane. Therefore, this conformal geometry preserves receptive fields and retinal illuminance, providing constructive properties for human vision (Turski, 2012).

Now, for each of the binocular system's AEs, the fixation point $F$ in the horizontal visual field defines the origin in the image plane and $F_{\infty}$ is projected to the point at infinity. The origin and the point at infinity are images of the fovea and the nodal point under stereographic projection, which identifies the spherical retina with the image plane and, therefore, they are opposite points in the Riemann sphere. I assume that $F$ and $F_{\infty}$ are opposite points on the horopter of the binocular system with AEs. This assumption, which is confirmed in this paper by geometric constructions supported with dynamic geometry software, provides us with a particularly simple theory of empirical horopters that is both biologically supported and geometrically precise, advancing the classic model of empirical horopters introduced by Ogle (1932). Surprisingly, both stereographic projection and the horopter were first introduced by Aguilonius in his Six Books of Optics published in 1613 .

The demonstration of the main results of horopteric conics, referred to as binocular conics, is constructive and, thus, making possible to design algorithms for modeling stable binocular vision in mobile robots.

Binocular Conics Construction. For the binocular system with AEs' orientations such that point $F_{\infty}$ is in the visual field, the horopteric curve's center is designated the midpoint $M$ of line segment $F F_{\infty}$. This means that for each point on the curve, there is another point on this curve diagonally opposite to it. Then, this curve, either an ellipse or a hyperbola, is fully specified by $F, F_{\infty}$ and the nodal points $N_{r}$ and $N_{l}$. For coplanar image planes, when the eyes are fixating at the abathic distance, the parallel to the image planes straight line horopter passes through the symmetrically fixated point. Further, when $F_{\infty}$ is at infinity, that is, when the image planes are parallel but not coplanar, the horopteric curve is a parabola. Each conic section's orientation is exactly given by the version angle (2).

Demonstration: The horopteric curves in the binocular system with AEs are binocular conics under the assumed point symmetry of the horopteric circles in Proposition 1, they are geometrically constructed and graphically visualized in GeoGebra (Figure 3). Because the construction involves the same steps for both hyperbolas and ellipses, I construct only a hyperbola. For a given position in which the eyes' image planes are nonparallel, the eyes' nodal points $N_{r 1}$ and $N_{l 1}$, fixation point $F_{1}$, and point $F_{1 \infty}$, are all shown in Figure 3A. Then, two additional points on the conics are constructed in Figure $\mathbf{3 A}$ by taking reflections of the nodal points about midpoint $M_{1}$ of line segment $F_{1} F_{1 \infty}$. These additional points, shown as $R_{1}$ and $S_{1}$, determine the conics. Shown in this panel, the conic section graphed in GeoGebra by taking any five of these six constructed points, is the same hyperbola. Symmetric fixation at the abathic distance has 
coplanar image planes and fixations obtained from the primary position when eyes rotated by the same angle have parallel, noncoplanar image planes. In the first case, the two horizontal lines passing through their respective nodal points and parallel to their respective image planes overlap. We can use any point that is different from nodal points on the overlapping lines, point $F_{a \infty}$ in Figure 3B, to obtain the midpoint between that point and $F_{a}$. Reflections of the nodal points about the midpoint, point $M_{a}$ in Figure 3B, provide us with two additional points $R_{a}$ and $S_{a}$ that are colinear with point $F_{a}$ and, thus, define the straight line horopter that is parallel to the image planes. For any fixation obtained from $F_{a}$ by the same rotation angle of both eyes, the resulting image planes are parallel but not coplanar and $F_{\infty}$ is at infinity. In the projective geometry framework, $F_{\infty}$ is represented by a family of lines parallel to the eyes' image planes and the conics are parabolas. One of these parabolas is constructed for fixation $F_{3}$ in Figure 3B as follows. First, midpoint $L$ of the line segment connecting $N_{r 3}$ and $F_{3}$ is obtained and the line in the visual plane through $L$ that is parallel to the image planed. This line intersects the line that passes through $N_{l 3}$ and is parallel to segment $N_{r 3} F_{3}$ at the point $M_{l}$. Then, point $R_{3}$ on the parabola we want to construct is obtained by reflecting $N_{l 3}$ about the point $M_{l}$. The same steps are repeated starting with line segment $N_{l 3} F_{3}$ to obtain points $K$ and $M_{r}$. These points then give the second point $S_{3}$ on the parabola by taking a reflection of $N_{r 3}$ about $M_{r}$. All steps in these constructions, for example, taking a reflection about a point, are done via dynamic geometry environments of the GeoGebra system. The proof of the conic sections' orientations, specified by the version angle (2), is given for the horopteric ellipse in Figure 3C. The rays passing through $F_{\infty}$ intersect at $18^{\circ}$. This is the vergence at fixation point $F_{0}$ that is obtained for the symmetric eyes' azimuthal rotation angles $\phi_{r 2}=12^{\circ}$ and $\phi_{l 2}=30^{\circ}$. The two bisecting lines at $F_{\infty}$ and $F_{0}$ intersect at the right angle, proving that the orientation of the ellipse is given by the angle $\omega=21^{\circ}$ equal to the version $\omega_{0}=21^{\circ}$. This proof also holds for hyperbolas and parabolas. Because the values of the eye's asymmetry parameters are chosen arbitrarily, this proof applies to any parameters chosen for the eyes. Thus, the link between the horopter's geometry and eye movements is established. This ends DEMONSTRATION.

The geometrical construction of binocular conics in GeoGebra allows the graphical simulation the horopteric conics' transformations from the movement of the fixation point in the visual plane. The computer simulation of horopteric conics' transformations is included in Supplement 2.

How are these intrinsic properties of the theory related to human binocular vision? The human brain functions in physical space and receives information carried by light that is centrally projected onto the eyes' retinae and transduced by photoreceptors into electrochemical signals. After initial processing by the retinal circuitry, this visual information is mainly sent to the primary visual cortex where it produces specific retino-cortical mappings and forms input to other cortical areas (Wandell et al., 2007). This immensely complex processing decodes the environment from retinal stimulation and creates a neural representation of space (Sereno and Lehky, 2011), our subjective visual space.

The newest computational modeling in neuroscience that incorporates bicentric perspective mapping of the $3 \mathrm{D}$ environment onto the retinae demonstrates that this mapping is fundamental to the tuning of retino-cortical neuronal processes and these process's corresponding aspects of perception (Bonnen et al., 2019). Although the tuning was specifically examined for $3 \mathrm{D}$ motion in the primate cortical area MT, the process of decoding the world from retinal stimulation in visuomotor cortical areas must be strongly affected by the geometry that links the environment to the sensory epithelium, regardless of whether nonhuman or human primates locomote or scan the environment while standing still. This geometric relationship constraining visual perception in my theory is the bicentric projective mapping between 3D space and the AEs' image planes, which are determining the horopter's shape.

\section{RETINAL CORRESPONDENCE}

In normal binocular vision, the foveae are corresponding elements. This means that the fixated point is perceived in one direction-the principal, or Cyclopean, direction. The horopter curve through the fixation point is the locus of spatial points that project to the retinal corresponding elements such that each point of the horopter is perceived in the same direction relative to the Cyclopean direction.

Based on the results obtained in Binocular Conics CONSTRUCTion, the straight line horopter shown in Figure 4, which passes through the fixation point $F_{a}$, is established by the image planes' coplanarity. The right AE's visual axis passes through its respective nodal point and intersects the retina at the fovea $f_{r}$ and the image plane at point $O_{r}$. The other visual axis passes, similarly, through the nodal point of the left eye before intersecting the retina at $f_{l}$ (the fovea) and the image plane at $O_{l}$. These $O_{r}$ and $O_{l}$ points are the binocular correspondence centers of the image planes.

A point $Q$ on the linear horopter projects to retinal corresponding points: $q_{r}$ in the right eye, $q_{l}$ in the left eye, and $Q_{r}$ and $Q_{l}$ on the respective image planes, also called the image planes' corresponding points. The corresponding points $q_{r}$ and $q_{l}$ are located at different distances from their respective foveae such that the asymmetric distribution of corresponding retinal points with respect to the foveae is the result of the eyes' asymmetry and the head's bilateral symmetry (Porter et al., 2001; Mosquera and Verma, 2016). However, from similar triangles, $\triangle Q F_{a} N_{r}$ and $\triangle Q_{r} O_{r} N_{r}$ for the right eye, and similar triangles, $\triangle Q F_{a} N_{l}$ and $\triangle Q_{l} O_{l} N_{l}$ for the left eye, we conclude that $\left|Q_{r} O_{r}\right|=\left|Q_{l} O_{l}\right|$. This line horopter and the head's bilateral symmetry is used here to define the binocular correspondence as follows.

Retinal Correspondence. Referring to Figure 4, let $O_{r}$ and $O_{l}$ be projection points of fixation point $F_{a}$ into the right and left image planes of AEs, respectively. Then, any two points $Q_{r}$ and $Q_{l}$ of the equal distance from, and on the same side of $O_{r}$ and $O_{l}$ project, through the nodal points $N_{r}$ and $N_{l}$, to the retinal 


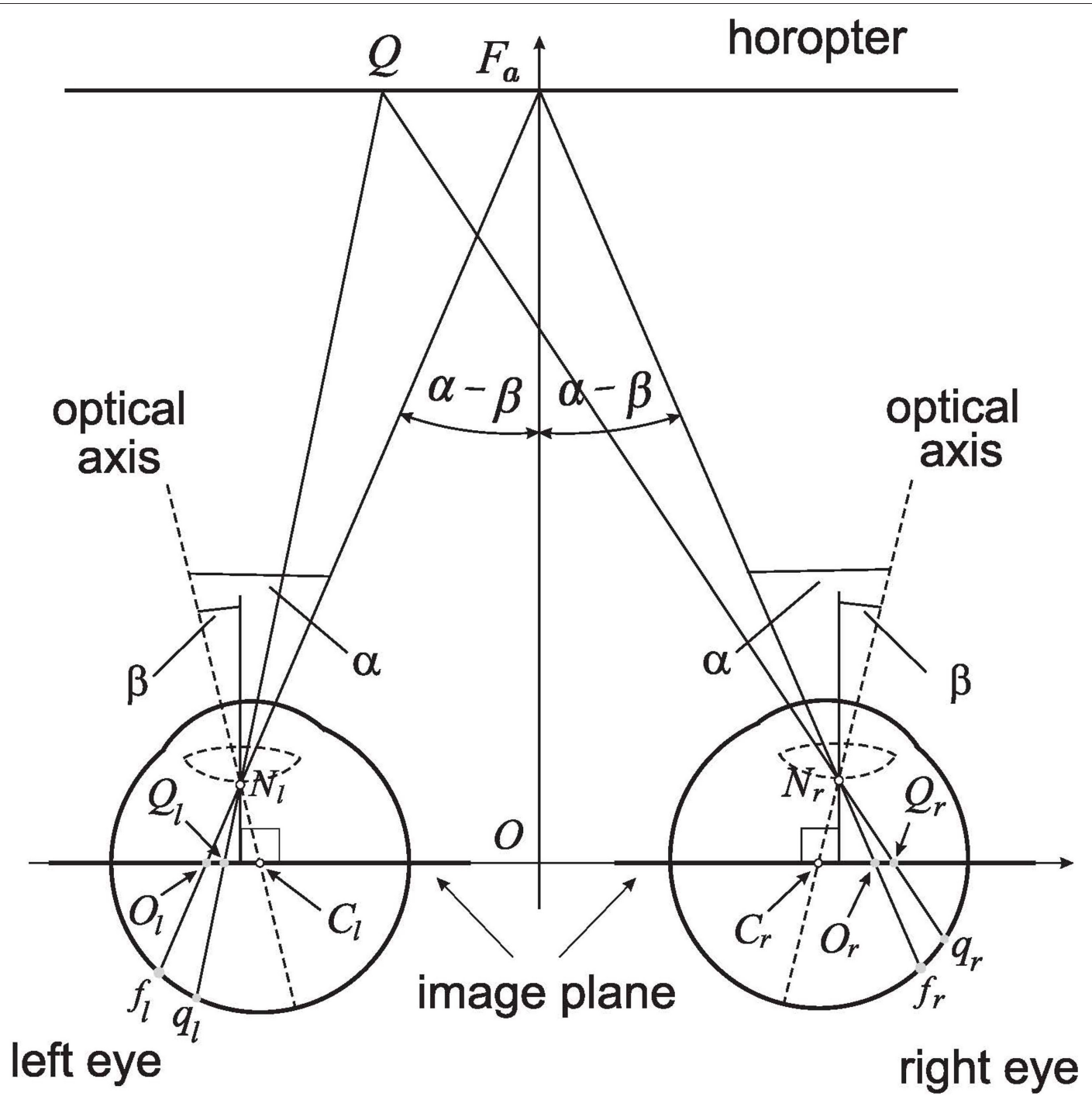

FIGURE 4 | The retinal correspondence's relation is formulated for the linear horopter at the abathic distance fixation $F_{a}$ in the binocular system with AEs. $F_{a}$ projects along the visual axes to the foveae $f_{r}$ and $f_{l}$ and the related image planes' optical centers $O_{r}$ and $O_{l}$. The point $Q$ projects to the retinal corresponding points $q_{r}$ and $q$ and their image planes' counterparts $Q_{r}$ and $Q_{l}$. The asymmetric distribution of retinal corresponding points covers, under the projections through the nodal points,

the symmetric distribution of related points on the image planes, which is proved in the text.

corresponding points $q_{r}$ and $q_{l}$ of unequal distance from the foveae $f_{r}$ and $f_{l}$, respectively.

This definition of the retinal correspondence, which is based on the geometric construction of binocular conics in the previous section, fully agrees with the retinal correspondence formulated in an ad-hoc way in Turski (2018). The abathic distance $d_{a}=$ $\left|O F_{a}\right|$ to the linear horopter at $F_{a}$ was obtained in Turski (2018). Here, the abathic distance is given in terms of asymmetry parameters, $\alpha$ and $\beta$, and interocular length, $2 a=\left|C_{r} C_{l}\right|$, in an equivalent but simplified form,

$$
d_{a}=\frac{a \cos (\alpha-\beta)+0.6 \sin \alpha}{\sin (\alpha-\beta)}
$$

where 0.6 is the distance in centimeters from the nodal point to the eye's rotation center. Then, using the average human values $2 a=6.5 \mathrm{~cm}, \alpha=5.2^{\circ}$, and $-0.4^{\circ} \leq \beta \leq 4.7^{\circ}$ in Equation (3), we obtain $34 \mathrm{~cm} \leq d_{a} \leq 380 \mathrm{~cm}$. However, in rare cases, the values of $\beta$ can approach more closely the value of $\alpha$, giving much larger value of $d_{a}$.

Further, for the average value of $\beta=3.3^{\circ}$, the abathic distance (3) is $99.61 \mathrm{~cm}$, a value consistent with the average value recorded in humans (Gibaldi et al., 2017). This distance is similar to the eye muscle's natural tonus resting position distance (Jaschinski et al., 2007), which serves as a zero-reference level for the eyes' convergence effort (Ebenholtz, 2001). Therefore, I refer to the position of the eyes fixating at the abathic-distance as the resting vergence position in order to distinguish it from the primary eyes' position. 
Each point on one of the binocular conics projects along the eyes' visual lines to the retinae of the AEs and defines one pair of corresponding points. However, only two pairs of points are used in the binocular conics' construction: the two foveae and the two points at infinity. All other corresponding retinal elements are established by the bicentric retinal projections of the horopter's points. The retinal correspondence is a well-defined concept only if the corresponding retinal points are independent of the binocular conics transformations when the fixation point moves in the visual plane. To verify this, in a computer simulation in the GeoGebra, available in Supplement 2, retinal corresponding points, $q_{r}$ and $q_{l}$, and the image plane's corresponding points, $Q_{r}$ and $Q_{l}$, are both determined by point $Q$ (cf. Figure 4) on the abathic distance line horopter and so remain corresponding when the eyes' position changes in the visual plane of fixations. Because this must be true for all pairs of retinal corresponding points, I conclude the following:

Remark 1. The Binocular correspondence's relationship introduced in RETINAL CORRESPONDENCE is well-defined.

The horopter's shape establishes a well-defined retinal correspondence. This theoretical relationship mirrors the one in human binocular vision in which retinal correspondence of normal binocular vision is specified by the two, corresponding, foveae such that all other corresponding retinal elements are then determined from laboratory measurements of the empirical horopter. However, the question of whether the corresponding retinal elements are fixed or not has remained undecided (Wick, 1991; Hillis and Banks, 2001).

In the theory presented here, binocular correspondence depends on the eye's asymmetry parameters. Therefore, the retinal correspondence can change when the asymmetry parameters change. Such changes can occur during refractive surgery. For example, to correct for refractive errors and achieve sharper vision, which is common for people with presbyopia, the crystalline lens are surgically replaced with an artificial IOL. Toric IOLs can also correct astigmatism caused by the shape of the cornea by adjusting the lens' orientation because they have different powers in different meridians. When a group of 333 patients were evaluated in Wang et al. (2019) for preoperative crystalline lens and postoperative IOL tilt, their IOL's tilt magnitude was found to have increased significantly by $1.2^{\circ} \pm 1.1^{\circ}$ compared to the preoperative crystalline lens tilt. I conclude from these results that postoperative change in the lens' tilt can be large enough to change the patient's empirical horopter's shape and the horopter's retinal correspondence. In the binocular system with asymmetric eyes, this change in the lens's tilt is modeled by the angle $\beta$ 's corresponding change.

\section{ANTHROPOMORPHIC BINOCULAR CONICS}

Figure 5 depicts the binocular conics given by the numerical method from Turski (2018) (dashed lines) and the geometric method developed in section 4 (solid lines) and drawn by GeoGebra's software for the average parameters observed in humans: $\alpha=5.2^{\circ}, \beta=3.3^{\circ}$, and ocular distance $2 a=6.5$ $\mathrm{cm}$. From the figure, we see that the hyperbolas for fixation $F_{1}$ and the ellipses for fixation $F_{2}$ obtained by both methods overlay each other nearly perfectly. However, the horopteric parabola for fixation $F_{3}$ on AIS differs from the tangent line to the VMC at $F_{3}$. Figure 5 shows that the difference between AIS (solid line through $F_{a}$ ) and VMC (dot-dashed line through $F_{3}$ ) should be insignificant to the perceptually important $90^{\circ}$ of the central visual field.

To find the difference between the AIS and VMC, I first note that the AIS can be well-approximated with a circle. In fact, using GeoGebra, I find that the AIS's approximation in the visual field's range of $\pm 45^{\circ}$ (cf. section 3) to 2 decimal places is the circle $x^{2}+(y-49.46)^{2}=(50.13)^{2}$. To find the equation of the VMC passing through $F_{3}$, I recall the exact geometric description of the VMC given in Turski (2016a): the center $(0, k)=(0, a /(2 \tan \eta)$ and the radius $R=a /(2 \sin \eta)$. Then, upon substituting $a=3.25$ $\mathrm{cm}$ and using the computed in GeoGebra value of $\eta=3.73^{\circ}$ at $F_{3}$, the VMC's equation is $x^{2}+(y-49.80)^{2}=(49.91)^{2}$. This verifies that the difference between the two circles is negligible.

In Figure 5, all angles are obtained in GeoGebra by the geometric method of this paper and displayed to an accuracy of 5 decimal points. The fixation point $F_{1}$ is obtained by rotations $\phi_{r}=9^{\circ}$ and $\phi_{l}=10^{\circ}$ from the resting vergence position and gives rise to the hyperbola constructed in section 4 . Version $\omega_{1}=$ $9.5^{\circ}$ gives the hyperbola's orientation while $F_{1}$ 's orientation angle is $\omega_{F_{1}}=9.50009^{\circ}$. The fixation point $F_{2}$ results from rotations by $\phi_{r}=-13^{\circ}$ and $\phi_{l}=-15^{\circ}$ and gives rise to the ellipse, again with an orientation specified by version $\omega_{2}=-14^{\circ}$ and the direction of $F_{2}$, which was given by azimuthal angles $\omega_{F_{2}}=$ $14.00061^{\circ}$. Rotations $\phi_{r}=\phi_{l}=-20^{\circ}$ from the resting vergence position gives the fixation point $F_{3}$ on AIS and version $\omega_{3}=$ $-20^{\circ}$ specifies the resulting parabola's symmetry axis's direction. The point on the corresponding VMC midway between the eyes provides the direction, $\omega_{F_{3}}=-20.00039^{\circ}$, of $F_{3}$. We see that the conics' orientations and the fixation points' directions differ by $<4 \mathrm{~s}$ of an arc. This insignificant difference allows me to place the Cyclopean eye at the same point on the corresponding VMC it was placed at in the binocular system with symmetric eyes (Turski, 2016a): midway between the eyes' centers.

We conclude from Figure 5 and the results in Turski (2018) that, for the anthropomorphic binocular system, the binocular conics in my theory are numerically close to the conic sections obtained in Ogle (1932) and Amigo (1965). I can therefore express the conic sections' parameter $H$ used in those studies in terms of the AE's parameters $\alpha$ and $\beta$. At the abathic distance, $H=2 a / d_{a}, 2 a=6.5 \mathrm{~cm}$ is the interocular separation and $d_{a}$ is the abathic distance to the fixation point given in Equation (3). Thus, $H$ can be expressed in terms of the eye's asymmetry parameters as follows:

$$
H=\frac{2 a \sin (\alpha-\beta)}{a \cos (\alpha-\beta)+0.6 \sin \alpha} .
$$

Because the subtense $\sigma_{a}=2(\alpha-\beta)$ at the abathic distance fixation is small (0.066 radians for the anthropomorphic parameters $\alpha=5.2^{\circ}, \beta=3.3^{\circ}$ ), we obtain the approximation 


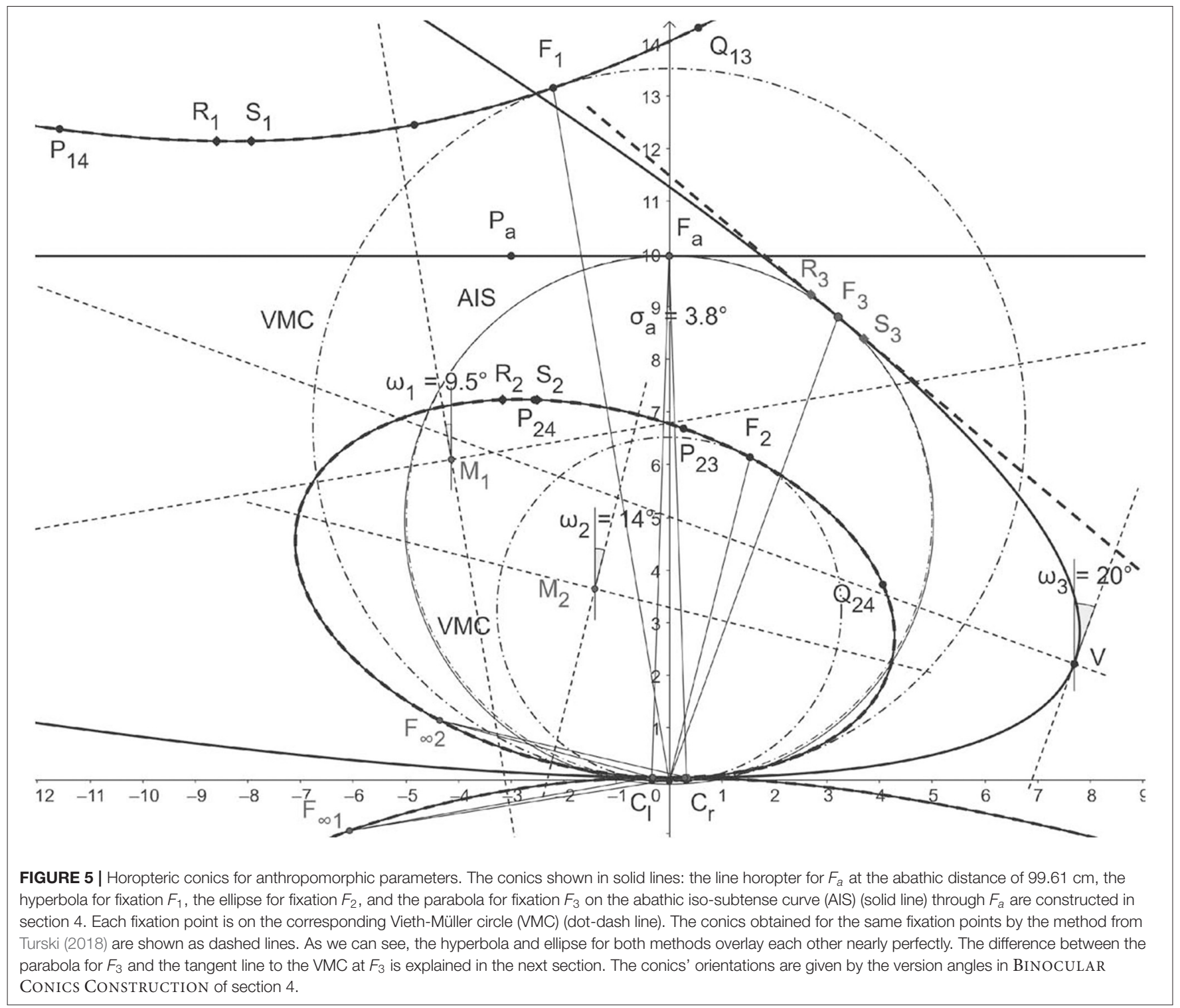

$H \approx 0.065$, which differs from $\sigma_{a}$ by approximately $0.001 \mathrm{rad}$. Moreover, for $\alpha=5.2^{\circ}$ and the range of $\beta$ 's values, $-0.4^{\circ}<$ $\beta<4.7^{\circ}$ (cf. section 2), I obtain the range of $H$ 's values in Equation (4) as follows: $0.01<H<0.19$. This result for H's values is consistent with Ogle's original estimation of $0<H<0.2$ for human subjects (Ogle, 1932, 1950). Ogle (1950) presented the values of $H$ calculated from the data of Helmholtz, Lau, and Libermann among many other researchers obtained in Nonius observations, which are in the range of his original values of $H$ reported in Ogle (1932). See also the relevant discussion in Shipley and Rawlings (1970). It is also consistent with the values estimated more recently in Schreiber et al. (2008) and Gibaldi and Banks (2019). However, these recent studies were more general by considering the Hering-Hillebrand deviation parameter $H$ and the Helmholtz shear, or the vertical horopter's backward inclination, that is not included in my study.

\section{BINOCULAR CONICS IN VISUAL PLANE}

A theory of horopteric circles in the binocular system with symmetric (reduced) eyes may be based on Euclidean geometry alone. But for a theory of horopteric conics in the binocular system with AEs, a framework of projective geometry is necessary. In projective geometry terms (Henle, 1997), the general conic equation given by the inhomogeneous quadratic polynomial $c(x, y)$,

$$
c(x, y)=A x^{2}+B x y+C y^{2}+D x+E y+F=0,
$$

is also expressed by the homogeneous quadratic form $C(X, Y, Z)=Z^{2} c(X / Z, Y / Z)$.

Although no more than five points on a conic are needed to find its equation, this straightforward task appears computationally unfeasible for binocular conics because the 
expressions for the points specifying a generic binocular conic are too complicated. To circumvent this limitation, I classify binocular conics in terms of discriminants of Equation (5) and analyze the classes of conics in the "general position" when the point of bifoveal fixation moves in the horizontal visual plane. The notion "general position" will be explained below in this section.

The conic (5) is degenerate if and only if its discriminant, i.e., the determinant $\Gamma$ of the symmetric matrix of its homogeneous quadratic polynomial, vanishes. Here,

$$
\Gamma=\operatorname{det}\left[\begin{array}{ccc}
A & B / 2 & D / 2 \\
B / 2 & C & E / 2 \\
D / 2 & E / 2 & F
\end{array}\right] .
$$

Then, for either degenerate or nondegenerate conics, its type is determined by the sign of the quadratic part of discriminant of Equation (6),

$$
\Delta=\operatorname{det}\left[\begin{array}{cc}
A & B / 2 \\
B / 2 & C
\end{array}\right]=A C-\frac{1}{4} B^{2} .
$$

The cases restricted to the real degenerate conics $\Gamma=0$ are: two intersecting lines $\Delta<0$, two parallel lines $\Delta=0$, and one point $\Delta>0$. The non-degenerate conics $\Gamma \neq 0$ are classified as follows: the conic is a hyperbola if $\Delta<0$, an ellipse if $\Delta>0$, and a parabola if $\Delta=0$ (see Reid, 1988).

The three conics are as follows: the hyperbola for the fixation $F_{1}$, the ellipse for the fixation $F_{2}$, and the parabola for the fixation $F_{3}$, shown in Figure 5, have the following equations and discriminants (7) obtained in the numerical simulations with GeoGebra for the calculated points in the constructions carried out for demonstration of BinOcular Conics CONSTRUCTION in section 4 .

1. Hyperbola's branch containing $F_{1}: 2.73 x^{2}+3.77 x y-8.22 y^{2}-$ $0.40 x+115.86 y-7.11=0 ; \Delta=-26$

2. Ellipse containing $F_{2}: 1.12 x^{2}+0.90 x y+2.81 y^{2}-0.12 x-$ $19.24 y+0.99=0 ; \Delta=2.9$

3. Parabola containing $F_{3}: 0.21 x^{2}+1.14 x y+1.56 y^{2}-0.14 x-$ $17.72 y+0.97=0 ; \Delta=-6 \times 10^{-7} \approx 0$

The discriminant value for the parabola, which should be 0 , is only approximated by -0.0000006 . This explains why the parabola was approximated in Turski (2018) by a straight line, whereas, here, it is given by the parabola (cf. Figure 5). In this theory, the parabola is built into the model of horopteric curves by way of construction. However, in the numerical simulation in Turski (2018), the conics' sensitivity near $\Delta=0$ allows us to see either an ellipse or hyperbola with the shape that resembles the tangent line near the fixation point. What could explain this?

Intuitively, the set of conics satisfying condition $\Gamma=0$ is negligible when compared to the set of conics satisfying $\Gamma \neq 0$ because the number of conics enumerated by $\{\Gamma=x, x \in R \backslash\{0\}\}$ is huge compared to conics enumerated by $\Gamma=0$. Similarly, when $\Gamma \neq 0$, the set of conics satisfying $\Delta=0$ is negligible when compared to the set of conics satisfying $\Delta \neq 0$. In mathematics, see Hazewinkel (1989) for example, the "general position" is a notion of genericity for geometric objects satisfying some special conditions that distinguishes them from all other geometric objects in a given collection. Thus, in the whole collection, the subcollection of objects in their general position is "massive," and the complementary set "meager," with its objects "negligible." Thus, only ellipses and hyperbolas are conics in the general position.

Now, after these preliminary remarks, I can analyze the binocular conics in the visual plane of bifoveal fixations. To this end, I note that the fixation points inside the AIS curve produce the eyes' positions such that $\eta=\phi_{r}-\phi_{l}>0$, while the eyes' positions with fixation points outside the AIS satisfy $\eta=\phi_{r}-\phi_{l}<0$. This simple property of the eyes' positions and the computer simulation of binocular conics lead to the following proposition about horopteric geometry in the visual plane:

Binocular Conics Transformation. If $\alpha>\beta$, then the AIS of a constant subtense of $2(\alpha-\beta)$ divides the visual plane into three distinct regions: (A) The fixation point $F$ on the AIS determines the horopter as a parabola if $F \neq F_{a}$, and a straight line if $F=F_{a}$. (B) The fixation point outside of the AIS specifies the branch of a horopteric hyperbola through this point, possibly degenerating into two intersecting lines at some of the fixation points. $(C)$ The fixation point in the binocular field inside of the AIS specifies a horopteric ellipse. On the other hand, in the monocular field inside the region enclosed by AIS, an ellipse can change into a hyperbola such that the sequence of transformed conics passes through the degenerate case of two parallel lines.

This classification of binocular conics transformations is demonstrated in a GeoGebra simulation for human-like binocular system parameters when the fixation point moves in the visual plane (Supplement 2).

This simulation provides the binocular conics "noisy" classifications given in terms of eye position, information that is available to the visual system. Thus, when the fixation point is moved in the visual plane, only the initial linear horopter and the evolution of subsequent conics in the general position can be observed. For example, the degenerate conics cases mentioned above in BINOCULAR CONICS TRANSFORMATION: the two intersecting lines and two parallel lines can only be inferred from observing neighboring conics in their general positions. The three typical cases of observed binocular conics: the straight line as the initial horopter at the abathic distance fixation, and the hyperbola and ellipse obtained in the simulation, are shown, respectively, in Figures 6A-C. Atypical cases of the simulation are also shown in Figures 6D-F. In these panels, the conic morphs through two parallel lines from ellipses to hyperbolas. Further, in Figure 6E, the conic shown is indeed a hyperbola, although its branches may appear in the figure to be parallel lines. Running several dozens of simulation sessions attests that this will only happen well outside the binocular region. In these three last panels, points where the visual axes intersect are near the eyeball in a space where fixations are prevented by human anatomy.

To summarize the conclusions of this section, both the perceived direction of the point being fixated on, i.e., the 


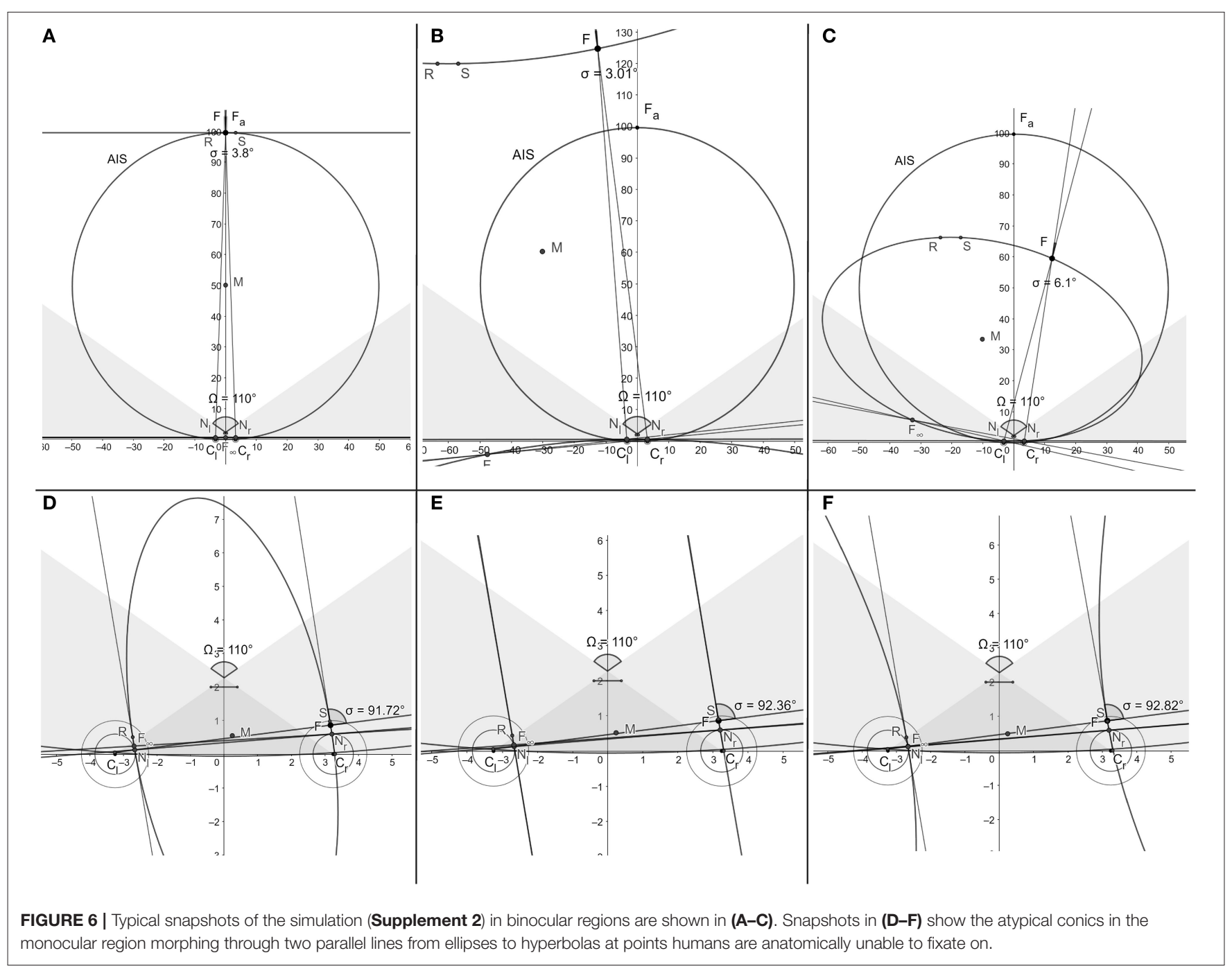

Cyclopean direction, and the binocular conic's orientation can be assumed to be entities specified by the version angle (2). This would mean that when the eyes rotate to change gaze in the horizontal plane, both the Cyclopean direction and the binocular conic undergo the same rotations by the version angle. But this would also imply that an object seen to be moving in a frontal line is really moving along the constantly changing horopters in the direction tangential to the instantaneous horopter curves. The curve traced out by the fixation point tracking this object is an iso-subtense curve. This implies that the rotations of both eyes during this pursuit are equal for the AIS passing through the resting vergence position, or that the rotations differ by a constant value along other iso-subtense curves in the horizontal plane. For eyes pursuing other object's trajectories, the difference in the two eyes' rotations is time dependent. For example, this is the case when the object in pursuit moves along the straight frontal line on a flat projection screen in what is a typical laboratory setting.

\section{DISCUSSION}

The horopter's significance in stereoscopic vision can be explained as follows. When a point in the visual plane lies in front of or behind the horopter curve containing the fixation point, the difference in the angles subtended on each retina between the image and the fovea's center defines retinal disparity. For each point on the horopter, there is maximum disparity for the single vision that defines Panum's fusional area around the horopter curve. In this region, non-corresponding retinal elements are fused to provide us with both single vision and the ability to see visual objects stereoscopically in depth from the observer's current point of fixation. Taking the difference in retinal disparities for a pair of points then provides us with the relative disparity used for our perception of 3D form. Objects outside Panum's area fall on widely disparate retinal areas and are seen as coming from two different visual directions, causing physiologic diplopia, or double vision. Here, with bicentric 
projective geometry and a novel model eye, I studied the basic concepts most useful to understanding stereopsic vision: retinal correspondence, horopters, and the Cyclopean axis.

\subsection{Retinal Correspondence and Geometric Horopters}

The geometry of longitudinal horopteric conics integrated with eye movements is constructed in the framework of bicentric perspective projections on the image planes of the AEs. The $\mathrm{AE}$ is a model eye that includes the eyeball's global asymmetry caused by the fovea's displacement from its posterior polethe main source of the eye's optical aberrations-and the crystalline lens' tilt that is countering some of these aberrations (Artal, 2014). The theory demonstrates that (i) the longitudinal horopteric curves for the binocular system with AEs are conic sections and (ii) the retinal correspondence obtained from the horopteric conics is a well-defined concept. Moreover, using this theory allows us to assert that the conics sections' branches, which pass through the fixation points and referred to as binocular conics, closely resemble empirical horopters obtained by laboratory measurements with the Nonius method (Ogle, 1950; Shipley and Rawlings, 1970). Until recently, there has been only one comprehensive model of empirical horopters and it was elaborated in Ogle (1932) and Amigo (1965) by an ad hoc introduced equation with a free parameter determined experimentally for each subject. The geometric theory developed here advances that classic model of Ogle and Amigo by establishing a physiologically motivated model of the empirical horopters integrated with the eyes' movements.

This theory accounts for the fundamental fact that the human visual system functions in physical space and acquires visual information by actively scanning the environment when we are awake. Incident light rays reflected from objects in a scene in 3D space are projected onto the unstable $2 \mathrm{D}$ retinae and neuronal processes activated in the visual and visuomotor cortical areas decode and interpret the scene's $3 \mathrm{D}$ properties. Therefore, any decoding of the environment's $3 \mathrm{D}$ properties from sensory information must be fundamentally constrained by the sensory organs' geometric relationship to the environment (Rokers et al., 2011; Bonnen et al., 2019) and modulated by the eyes' movements (Hejtmancik et al., 2016).

The conceptual framework used here in constructing horopteric conics for the binocular system with AEs not only provides a biologically based model that reproduces empirical horopters, it also provides a framework for the theory of geometric horopters developed in Turski (2016a) for the binocular system with the symmetric (reduced) model eye. This result is proved here in Proposition 1 for any position of the nodal point between the eye's pupil and its center of rotation, including, of course, the location of the anatomical nodal point. Thus, three qualitatively different theories of the geometric horopters, including the theory of the VMC, are constructed here in the framework of bicentric projections. The three theories are briefly compared below in the order of their respective model eye's anatomical fidelity.
The first model is a special case of the symmetric model eye in which the nodal point is taken to coincide with the eye's rotation center. Proposed almost two centuries ago, the resulting horopter curves are the iso-vergence circles, or VMCs, each passing through the fixation point and connecting the eyes' rotation centers. When the eyes fixate on points along the VMC, the eyes' rotation centers do not move. This means that the VMC and the vergence value also do not change when the eyes fixate on points along the VMC. Further, relative disparity becomes independent of eye position in this model eye (Turski, 2016a). This model-dependent constancy is a consequence of incorrectly locating the nodal point at a position that is not its anatomical position.

The second model is the symmetric model eye with a nodal point located $0.6 \mathrm{~cm}$ anterior to the eyeball's rotation center as required by the eye's anatomy. Its horopter curves consist of a family of circles passing through the fixation point and connecting the nodal points (Turski, 2016a). For a constant vergence value, these horopteric circles are parameterized by specific fixation points on the binocularly visible part of the VMC and intersect at the VMC's point of symmetric convergence. Relative disparity, in this model, depends on eye movement and its changes are always within the binocular acuity limits for fixational eye movements (Wilcox and Harris, 2010). Regardless of this result, relative disparity is often assumed independent of the eyes' position. I hypothesized in Turski (2016a) that the size and shape changes perceived during fixational eye movements may not only provide perceptual benefits, such as breaking camouflage, but may also provide the aesthetic benefit of stereopsis (Ponce and Born, 2008).

The third binocular system, with AEs of the highest anatomical fidelity, is the subject of this paper. In this system, the geometric horopters are binocular conics resembling empirical horopters and their orientation is exactly specified by the version angle, giving this angle a new significant meaning in biological vision. On the other hand, if the Cyclopean axis is defined from the midpoint on the VMC's arc connecting the eyes' centers of rotation, the same way as it was defined in the binocular system with symmetric eyes, its direction given by the azimuthal angle provides the best approximation of the vergence angle in the human's binocular system; the difference between the Cyclopean eye direction and the binocular conic orientation given by the version is on the order of a few seconds of an arc (Figure 5).

Although the VMCs and empirical horopters have different geometries, the VMC is often identified with the longitudinal horopter. The VMC does provide a good approximation for the empirical horopter near the fixation point, but the difference in their geometries is significant in the periphery. A small object peripherally located on the VMC will have zero disparity with respect to this horopter model, but it will have a nonzero disparity with respect to the binocular conics that approximate well the empirical horopters over the whole visual field. Visually guided saccades intercepting a peripherally viewed object will be well off the target if programmed in terms of VMC's disparity. Although the simplicity of the VMC makes it useful in some numerical aspects of visuomotor research, its approximation of both geometric and empirical horopters is a crucial condition 
that should always be emphasized in order to avoid its, currently frequent, mischaracterizations.

Further, it is suggested in Sprague et al. (2015) that the shape of the longitudinal horopter is a result of the visual system allocating resources according to natural disparity statistics for binocular correspondence matches. Although the horopter's shape can support these statistics, my theory instead asserts that the shape of empirical horopters is caused primarily by the misaligned optical elements modeled by the AE. In fact, in healthy eyes, the fovea is displaced from the eyeball's posterior pole and the cornea and the crystalline lens are tilted relative to each other (Chang et al., 2007; Holladay, 2007; Schaeffel, 2008). The crystalline lens' tilt cancels out some of the aberrations caused by foveal displacement and the cornea's asphericity and produces nearly aberration-free perception near the visual axis (Tabernero et al., 2007; Artal, 2014). Then, the adaptation to the natural environment's visual statistics can be achieved through the binocular eye's movements (Canessa et al., 2017; Gibaldi and Banks, 2019).

\subsection{Binocular Conics and Eye Movement}

The fovea, which has the highest visual acuity on the retina, subtends only a two-degree visual angle. To prevent diplopia, a saccade must quickly direct the eyes' foveae toward the objectin what is called the conjugate eye movements because the eyes are rotating in the same direction. Saccades usually need to be corrected by a vergence-the disjunctive eye movements as they rotate in opposite directions, and then the foveae must be held precisely aligned on the object (Masson et al., 2001; Maxwell and Schor, 2006). This corrective vergence movement, or motor fusion, adjusts the eyes' alignment to maintain sensory fusion (Schor, 1979; Liversedge et al., 2006).

Cortical activity derived from bicentric perspective retinal stimulation must, therefore, be modulated by the eyes' movements (Hejtmancik et al., 2016). The size and direction of the adjustment is given by the binocular disparity between the currently viewed object and the next one to be viewed. Thus, the concept of retinal corresponding elements is not only fundamental to single vision and stereopsis, it is also important in the binocular coordination of the eyes' movements. Understanding how the eyes' movements are controlled by the visuomotor processes and how they affect the precise correspondence of the retinal elements remains uncertain (Waitzman, 2016).

Moreover, during natural viewing, the human eye's rotational speeds during saccades are as fast as $700^{\circ} / \mathrm{s}$, with an acceleration exceeding $20,000^{\circ} / s^{2}$ (Waitzman, 2016). Saccadic eye movements are performed about 3-4 times/s, meaning that visual information is mainly acquired by the brain during 34 brief fixations within a second. In addition, we are not only able to execute smooth pursuit eye movements that keep the foveae focused on a slowly moving object up to $100^{\circ} / \mathrm{s}$; we also employ a combination of smooth pursuit and saccades to track an object moving unpredictably or moving faster than $30^{\circ} / \mathrm{s}$ (Westheimer, 1954; Meyer et al., 1985). By stabilizing the tracked object's image on the fovea, smooth pursuit eye movements (SPEMs) superimpose additional motion on the retinal images of the stationary background and on the moving objects.
For example, the consequences of the saccadic eye movements' high speed and acceleration markedly restricts the use of visual information between fixations. Therefore, the basic feature underlying natural viewing is the occurrence of intricate dynamic disparity that is then processed to maintain our clear vision that appears continuous and stable. In this regard, my theory provides the binocular conics' transformations by integrating the binocular conic's geometry with the eyes' changing position in the horizontal visual plane of bifoveal fixations, therefore extending my work on modeling the monocular vision stability in Turski $(2010,2016 b)$ to the binocular framework.

The kinematics of visually guided eye movements is constrained by Listing's law, which involves the primary eye position in this law's formulation. In its typical version, which originally applied to a single eye's rotation, when the eye fixates on a target at optical infinity, Listing's law asserts that, with the head upright and stationary, there is an eye position called the primary position such that any other eye orientation can be reached by a single eye rotation about the axis in the plane perpendicular to the eye's primary direction. This plane is known as Listing's plane. Consequently, during eye movements that obey Listing's law (e.g., saccades and smooth pursuit), the eyeball assumes a unique torsion, or a rotation about the line of regard, for each eye orientation (Crawford et al., 2003). In my study, all eye movements are constrained to rotate about the vertical axis such that the torsion specified by Listing's law is always zero.

Further, when both eyes are constrained to fixate binocularly during the eyes' rotations, binocular extension of Listing's law, known as L2, applies to the eyes' positions. L2 asserts that during convergence, the eyes' rotation axes still remains confined to a plane for each vergence angle; however, as the eyes converge, these planes rotate relative to Listing's planes temporally and roughly symmetrically (Bruno and van den Berg, 1997; Tweed, 1997; Somani et al., 1998).

My theory specifies the AEs' primary position at the abathic distance fixation, or the resting vergence position, to allow for the physiologically motivated replacement of the imprecise primary eye position as follows. In the absence of visual cues, the eyes' gaze shifts to the eyes' natural tonus resting vergence position, which serves as a zero-reference level for convergence effort (Ebenholtz, 2001). In fact, the average natural tonus resting vergence distance for the forward gaze is of the same value as the human's average abathic distance, which also agrees with the abathic distance for the average anthropomorphic parameters of the AE. Moreover, though both the tonus resting vergence distance and the abathic distance vary from about $40 \mathrm{~cm}$ to optical infinity across subjects, they are reliable parameters within a subject (Owens and Leibowitz, 1980). Further, the resting vergence position is supported by recent results demonstrating that Listing's law and kinematics related to Listing's law are implemented peripherally and by the oblique extraocular muscles (EOMs) mechanism rather than centrally (Demer, 2006). In fact, these EOM's forces are indispensable to 3D modeling of eye movements and are responsible for the mechanical equilibrium of the eye suspended in resting vergence position (Gao et al., 2014). Moreover, the resting vergence position's change with 
lowered and elevated gaze (Heuer and Owens, 1989) agrees with the vertical horopter's backward inclination and its effect on perception (Amigo, 1974; Grove et al., 2001; Schreiber et al., 2008).

The above discussion strongly support my choice of the resting vergence position at the abathic distance of bifoveal fixation to replaces the eyes' primary position that essentially applies only to a single eye, but is often stated as describing both eyes fixating at optical infinity with an obvious lack of precision. This could be the reason that despite theoretical importance of the eyes' primary position, its precise formulation and neurophysiological significance remain elusive (Hess and Thomassen, 2014).

The theory of binocular conics constructed here needs to be further extended with the vertical component and integrated with $3 \mathrm{D}$ eye movements. This extension to a full framework of bicentric perspective (Koenderink, 1992) will inevitably introduce a host of geometric difficulties. For example, the visual line in the $\mathrm{AE}$ model that passes through the fovea, the optical node, and the fixation point differs, because of the fovea's displacement from the posterior pole of the eyeball, from the line of regard, or fixation axis, connecting the eye's rotation center with the fixation point. One of the questions this introduces is how to model the rotation of the visual axis by the eye's torsion around the line of regard because a rotation complicates the control of the binocular eyes' alignments in near-vision conditions. Moreover, the extension of Listing's law that applies when the eyes start rotating from their tertiary position, the so-called half-angle rule, was not needed here, but will be indispensable when my theory is extended to $3 \mathrm{D}$ rotations because this extensions requires the use of angular velocity rather than rotation axes (Tweed et al., 1990).

Also specific to 3D kinematics of eye-head bifoveal fixations is either the Listing's plane's tilt or Listing's plane geometry change to a twisted surface. The Listing's plane's geometry changes have been analytically modeled as the effect of the alignment maximization method (Chen et al., 2019). Further, it has been proposed in Ghosh et al. (2011, 2012) that the crystalline lens' horizontal tilt and the eye's axial length change during a $25^{\circ}$ downward gaze with binocular fixations at the $0.2 \mathrm{D}$

\section{REFERENCES}

Amigo, G. (1965). The mathematical treatment of horopter data obtained with the eyes in asymmetric convergence. Opt. Acta 12, 305-315. doi: $10.1080 / 713817940$

Amigo, G. (1974). A vertical horopter. Opt. Acta 21, 277-292. doi: $10.1080 / 713818889$

Artal, P. (2014). Optics of the eye and its impact in vision: a tutorial. Adv. Opt. Photon. 6, 340-367. doi: 10.1364/AOP.6.000340

Bonnen, K., Czuba, T. B., Whritner, J. A., Kohn, A., Huk, A. C., and Cormack, L. K. (2019). Binocular viewing geometry shapes the neural representation of the dynamic three-dimensional environment. Nat. Neurosci. 23, 113-121. doi: 10.1038/s41593-019-0544-7

Bruno, P., and van den Berg, A. (1997). Relative orientation of primary positions of the two eyes. Vis. Res. 37, 935-947. doi: 10.1016/S0042-6989(96)00219-2 and 2.5 D accommodative states for near-visual tasks. Although recent results in Liu and Thibos (2017) and Lu et al. (2020) support this proposition, the theory regarding the physiological mechanism of accommodation is still incomplete. It appears that the crystalline lens can change its tilt due to a loosening of the zonule-the fiber band attached to the lens that changes its curvature during accommodation-and gravity (Radhakrishnan and Charman, 2007), and careful modeling of this and other accommodative mechanisms may contribute to a fuller understanding of the origins of the elusive presbyopic changes (Charman, 2008). Thus, modeling physiological mechanisms underlying stereoscopic vision should include not only the eye's optical asymmetry but also the tilted, accommodating crystalline lens.

\section{DATA AVAILABILITY STATEMENT}

The original contributions presented in the study are included in the article/Supplementary Material, further inquiries can be directed to the corresponding author/s.

\section{AUTHOR CONTRIBUTIONS}

The author confirms being the sole contributor of this work and has approved it for publication.

\section{ACKNOWLEDGMENTS}

I acknowledge the reviewers' very helpful comments. I thank Alice Turski for her editing that has made this manuscript much easier to read. This manuscript has been released as a pre-print at BioRxiv (Turski, 2020).

\section{SUPPLEMENTARY MATERIAL}

The Supplementary Material for this article can be found online at: https://www.frontiersin.org/articles/10.3389/fnins. 2020.555965/full\#supplementary-material

Canessa, A., Gibaldi, A., Chessa, M., Fato, M., Solari, F., and Sabatini, S. P. (2017). A dataset of stereoscopic images and ground-truth disparity mimicking human fixations in peripersonal space. Sci. Data 4, 1-16. doi: 10.1038/sdata. 2017.34

Chang, Y., Wu, H.-M., and Lin, Y.-F. (2007). The axial misalignment between ocular lens and cornea observed by MRI (i) at fixed accommodative state. Vis. Res. 47, 71-84. doi: 10.1016/j.visres.2006.09.018

Charman, W, N. (2008). The eye in focus: accommodation and presbyopia. Clin. Exp. Optomet. 91, 207-225. doi: 10.1111/j.1444-0938.2008.00256.x

Chen, B., Fielding, J., and Chung, H. (2019). An alignment maximization method for the kinematics of the eye and eyehead fixations. Vis. Res. 158, 58-71. doi: 10.1016/j.visres.2019.01.013

Crawford, J., Martinez-Trujillo, J., and Klier, E. (2003). Neural control of threedimensional eye and head movements. Curr. Opin. Neurobiol. 13, 655-662. doi: 10.1016/j.conb.2003.10.009 
Demer, J. L. (2006). Current concepts of mechanical and neural factors in ocular motility. Curr. Opin. Neurobiol. 19, 4-13. doi: 10.1097/01.wco.0000198100.87670.37

Ebenholtz, S. M. (2001). Oculomotor Systems and Perception. Cambridge: Cambridge University Press. doi: 10.1017/CBO9780511529795

Gao, Z., Guo, H., and Chen, W. (2014). Initial tension of the human extraocular muscles in the primary eye position. J. Theor. Biol. 353, 78-83. doi: 10.1016/j.jtbi.2014.03.018

Ghosh, A., Collins, M. J., Read, S. A., and Davis, B. A. (2012). The influence of downward gaze and accommodation on ocular aberrations over time. Invest. Ophthalmol. Vis. Sci. 53, 6465-6471. doi: 10.1167/iovs.12-9973

Ghosh, A., Collins, M. J., Read, S. A., Davis, B. A., and Iskander, D. (2011). The influence of downward gaze and accommodation on ocular aberrations over time. J. Vis. 11, 1-13. doi: 10.1167/11.10.17

Gibaldi, A., and Banks, M. S. (2019). Binocular eye movements are adapted to the natural environment. J. Neurosci. 39, 2877-2888. doi: 10.1523/JNEUROSCI.2591-18.2018

Gibaldi, A., Canessa, A., and Sabatini, S. P. (2017). The active side of stereopsis: fixation strategy and adaptation to natural environments. Sci. Rep. 7, 1-18. doi: $10.1038 /$ srep 44800

Grove, P. M., Kaneko, H., and Ono, H. (2001). The backward inclination of a surface defined by empirical corresponding points. Perception 30, 411-429. doi: $10.1068 / \mathrm{p} 3091$

Guitton, D., and Volle, M. (1987). Gaze control in humans: eye-head coordination during orienting movements to targets within and beyond the oculomotor range. J. Neurophysiol. 58, 427-459. doi: 10.1152/jn.1987.5 8.3.427

Hazewinkel, M. (1989). Encyclopaedia of Mathematics, Vol. 4. Dordrecht; Boston; London: Kluwer. doi: 10.1007/978-94-009-5997-2

Hejtmancik, J., Cabrera, P., Chen, Y., M'Hamdi, O., and Nickerson, J. (2016). "Vision," in Conn's Translational Neuroscience, ed P. Conn (London; San Diego, CA; Cambridge; Oxford: Academic Press), 399-438. doi: 10.1016/B978-0-12-802381-5.00031-2

Henle, M. (1997). Modern Geometries: The Analytic Approach. Upper Saddle River, NJ: Prentice Hall.

Hess, B. J. M., and Thomassen, J. S. (2014). Kinematics of visually-guided eye movements. PLoS ONE 9:e95234. doi: 10.1371/journal.pone.0095234

Heuer, H., and Owens, D. A. (1989). Vertical gaze direction and the resting posture of the eyes. Perception 18, 363-377. doi: 10.1068/p180363

Hillis, J. M., and Banks, M. S. (2001). Are corresponding points fixed? Vis. Res. 41, 2457-2473. doi: 10.1016/S0042-6989(01)00137-7

Holladay, J. T. (2007). Quality of Vision: Essential Optics for Cataract and Refractive Surgeon. Thorofare, NJ: Slack Inc.

Jaschinski, W., Jainta, S., Hoormann, J., and Walper, N. (2007). Objective vs subjective measurements of dark vergence. Ophthal. Physiol. Opt. 27, 85-92. doi: $10.1111 / j .1475-1313.2006 .00448 . x$

Jaschinski-Kruza, W. (1991). Eyestrain in VDU users: Viewing distance and the resting position of ocular muscles. Hum. Factors 33, 69-83. doi: $10.1177 / 001872089103300106$

Koenderink, J. (1992). "Fundamentals of bicentric perspective," in Future Tendencies in Computer Science, Control and Applied Mathematics, Vol. 653, Lecture Notes in Computer Science, eds A. Bensoussan and J. P. Verjus (Berlin, Heidelberg: Springer), 233-251. doi: 10.1007/3-540-56320-2_62

Kowler, E. (2011). Eye movements: the past 25years. Vis. Res. 51, 1457-1483. doi: 10.1016/j.visres.2010.12.014

Liu, T., and Thibos, L. (2017). Variation of axial and oblique astigmatism with accommodation across the visual field. J. Vis. 17, 1-23. doi: 10.1167/ 17.3.24

Liversedge, S. P., White, S. J., Findlay, J. M., and Rayner, K. (2006). Binocular coordination of eye movements during reading. Vis. Res. 46, 2363-2374. doi: 10.1016/j.visres.2006.01.013

Lu, Q., He, W., Qian, D., lu, Y., and Zhu, X. (2020). Measurement of crystalline lens tilt in high myopic eyes before cataract surgery using swept-source optical coherence tomography. Eye Vis. 7:14. doi: 10.1186/s40662-020-00176-5

Martinez-Conde, S., Macknik, S. L., and Hubel, D. H. (2004). The role of fixational eye movements in visual perception. Nat. Rev. Neurosci. 5, 229-240. doi: $10.1038 / \mathrm{nrn} 1348$
Masson, G., Busettini, C., Yang, D.-S., and Miles, F. (2001). Short-latency ocular following in humans: sensitivity to binocular disparity. Vis. Res. 41, 3371-3387. doi: 10.1016/S0042-6989(01)00029-3

Maxwell, J. S., and Schor, C. M. (2006). The coordination of binocular eye movements: vertical and torsional alignment. Vis. Res. 46, 3537-3548. doi: 10.1016/j.visres.2006.06.005

Meyer, C. H., Lasker, A. G., and Robinson, D. A. (1985). The upper limit of human smooth pursuit velocity. Vis. Res. 25, 561-563. doi: 10.1016/0042-6989(85)90160-9

Mosquera, S., and Verma, S. (2016). Bilateral symmetry in vision and influence of ocular surgical procedures on binocular vision: a topical review. J. Optomet. 9 , 219-230. doi: 10.1016/j.optom.2016.01.005

Needham, T. (2002). Visual Complex Analysis. New York, NY: Clarendon Press.

Ogle, K. N. (1932). An analytical treatment of the longitudinal horopter; its measurement and application to related phenomena, especially to the relative size and shape of the ocular images*. J. Opt. Soc. Am. 22, 665-728. doi: 10.1364/JOSA.22.000665

Ogle, K. N. (1950). Researches in Binocular Vision. Philadelphia, PA: WB Saunders.

Owens, D. A., and Leibowitz, H. W. (1980). Accommodation, convergence, and distance perception in low illumination. Optomet. Vis. Sci. 57, 540-550. doi: 10.1097/00006324-198009000-00004

Ponce, C. R., and Born, R. T. (2008). Stereopsis. Curr. Biol. 18, R845-R850. doi: $10.1016 /$ j.cub.2008.07.006

Porter, J., Guirao, A., Cox, I. G., and Williams, D. R. (2001). Monochromatic aberrations of the human eye in a large population. J. Opt. Soc. Am. A 18, 1793-1803. doi: 10.1364/JOSAA.18.001793

Radhakrishnan, H., and Charman, W. (2007). Changes in astigmatism with accommodation. Ophthal. Physiol. Opt. 27, 275-280. doi: 10.1111/j.1475-1313.2007.00474.x

Reid, M. (1988). Undergraduate Algebraic Geometry. Cambridge; New York, NY; Melbourne, VIC: Cambridge University Press. doi: 10.1017/СBO9781139163699

Rokers, B., Czuba, T. B., Cormack, L. K., and Huk, A. C. (2011). Motion processing with two eyes in three dimensions. J. Vis. 11, 1-19. doi: 10.1167/11.2.10

Schaeffel, F. (2008). Binocular lens tilt and decentration measurements in healthy subjects with phakic eyes. Invest. Opthalmol. Vis. Sci. 49, 2216-2222. doi: $10.1167 /$ iovs.07-1022

Schor, C. M. (1979). The relationship between fusional vergence eye movements and fixation disparity. Vis. Res. 19, 1359-1367. doi: 10.1016/0042-6989(79)90208-6

Schreiber, K. M., Hillis, J. M., Filippini, H. R., Schor, C. M., and Banks, M. S. (2008). The surface of the empirical horopter. J. Vis. 8, 1-20. doi: 10.1167/8.3.7

Sereno, A. B., and Lehky, S. R. (2011). Population coding of visual space: comparison of spatial representations in dorsal and ventral pathways. Front. Comput. Neurosci. 4:159. doi: 10.3389/fncom.2010.00159

Shipley, T., and Rawlings, S. (1970). The nonius horopter-I. History and theory. Vis. Res. 10, 1225-1262. doi: 10.1016/0042-6989(70)90039-8

Somani, R. A., Desouza, J. F., Tweed, D., and Vilis, T. (1998). Visual test of listing's law during vergence. Vis. Res. 38, 911-923. doi: 10.1016/S0042-6989(97)00228-9

Sprague, W. W., Cooper, E. A., Tošić, I., and Banks, M. S. (2015). Stereopsis is adaptive for the natural environment. Sci. Adv. 1, 1-17. doi: $10.1126 /$ sciadv. 1400254

Tabernero, J., Benito, A., Alcón, E., and Artal, P. (2007). Mechanism of compensation of aberrations in the human eye. J. Opt. Soc. Am. A 24, 3274-3283. doi: 10.1364/JOSAA.24.003274

Turski, J. (2010). Robotic vision with the conformal camera: Modeling perisaccadic perception. J. Robot. 2010:130285. doi: 10.1155/2010/130285

Turski, J. (2012). Imaging With the Conformal Camera, Vol, II. Las Vegas, NV: IPCVIPR; CSREA Press.

Turski, J. (2016a). On binocular vision: the geometric horopter and cyclopean eye. Vis. Res. 119, 73-81. doi: 10.1016/j.visres.2015.11.001

Turski, J. (2016b). Modeling active vision during smooth pursuit of a robotic eye. Electron. Imaging 2016, 1-8. doi: 10.2352/ISSN.2470-1173.2016.10.ROBVIS-391

Turski, J. (2016c). The conformal camera in modeling active binocular vision. Symmetry 8, 1-22. doi: 10.3390/sym8090088 
Turski, J. (2018). Binocular system with asymmetric eyes. J. Opt. Soc. Am. A 35, 1180-1191. doi: 10.1364/JOSAA. 35.001180

Turski, J. (2020). A geometric theory integrating human binocular vision with eye movement. BioRxiv 1-20. doi: 10.1101/2020.09.03.280248

Tweed, D. (1997). Visual-motor optimization in binocular control. Vis. Res. 37, 1939-1951. doi: 10.1016/S0042-6989(97)00002-3

Tweed, D., Cadera, W., and Vilis, T. (1990). Computing three-dimensional eye position quaternions and eye velocity from search coil signals. Vis. Res. 30, 97-110. doi: 10.1016/0042-6989(90)90130-D

Waitzman, D. (2016). "Oculomotor systems and control," in Conn's Translational Neuroscience, ed P. Conn (London; San Diego, CA; Cambridge; Oxford: Academic Press), 439-465. doi: 10.1016/B978-0-12-802381-5.00032-4

Wandell, B. A., Dumoulin, S. O., and Brewer, A. A. (2007). Visual field maps in human cortex. Neuron 56, 366-383. doi: 10.1016/j.neuron.2007. 10.012

Wang, L., de Souza, R., Weikert, M., and Koch, D. (2019). Evaluation of crystalline lens and intraocular lens tilt using a swept-source optical coherence tomography biometer. J. Catar. Refract. Surg. 45, 35-40. doi: $10.1016 /$ j.jcrs.2018.08.025
Westheimer, G. (1954). Eye movement responses to a horizontally moving visual stimulus. Arch. Ophthalmol. 52, 932-941. doi: 10.1001/archopht.1954.00920050938013

Wick, B. (1991). Stability of retinal correspondence in normal binocular vision. Optomet. Vis. Sci. 68, 146-158. doi: 10.1097/00006324-199102000-00011

Wilcox, L., and Harris, J. (2010). "Fundamentals of stereopsis," in Encyclopedia of the Eye. Vol. 2, eds D. Dartt, J. Besharse, and R. Dana (New York, NY: Academic Press), 201-213. doi: 10.1016/B978-0-12-374203-2.00237-2

Conflict of Interest: The author declares that the research was conducted in the absence of any commercial or financial relationships that could be construed as a potential conflict of interest.

Copyright (๑) 2020 Turski. This is an open-access article distributed under the terms of the Creative Commons Attribution License (CC BY). The use, distribution or reproduction in other forums is permitted, provided the original author(s) and the copyright owner(s) are credited and that the original publication in this journal is cited, in accordance with accepted academic practice. No use, distribution or reproduction is permitted which does not comply with these terms. 